\title{
LAS FORMAS DE CONOCIMIENTO EN LA ENSEÑANZA. ANÁLISIS DE CLASES DE MATERIAS PEDAGÓGICAS EN UN INSTITUTO DE FORMACIÓN DOCENTE DE LA CIUDAD DE ROSARIO
}

\author{
Martín Acosta (Instituto Superior de Educación Física № 11, \\ Instituto de Educación Superior $\mathrm{N}^{\circ} 24$, \\ Instituto de Educación Superior $\left.\mathrm{N}^{\circ} 16\right)^{*}$ \\ martinracosta@yahoo.com \\ Ma. Elisa Welti (Universidad Nacional de Rosario)** \\ elisawelti@hotmail.com
}

Recibido: 30/07/2012 Aceptado: 22/12/2012

\section{Resumen}

La reseña de esta tesina defendida en el año 2010, presenta cuestiones que atraviesan cotidianamente a los profesores de Cs. de la Educación que trabajan en Institutos de Formación Docente. La motivación de indagar sobre este tema surge de la escasa bibliografía que aborda y profundiza esta modalidad. La intención entonces, fue conocer como se pone en juego el conocimiento en las clases escolares. A priori, se fue al campo con muchas sensaciones y supuestos que fueron desglosándose a medida que la investigación fue transcurriendo en el tiempo y en su forma. Se pensó en abordar cuáles serían los recorridos que se producen en las clases para lograr que el conocimiento surja dentro de ella, qué tipo de conocimiento se pone en juego, qué sucede con el conocimiento cuando se lo enseña o que propone el docente cuando enseña. Comprender las formas de conocimiento de la enseñanza que se pusieron en juego en las clases dictadas por los profesores del Instituto observados, ayudó también a analizar y reflexionar la propia labor docente cotidiana, y sobre todo, reconocer senderos, para mediar correctamente en la posibilidad de que los alumnos comprendan y aprendan los contenidos que se "juegan" en el aula.

* Licenciado y Profesor en Ciencias de la Educación. Facultad de Humanidades y Artes. Universidad Nacional de Rosario. Maestrando en Educación Superior Universitaria. Universidad Nacional de Rosario.

** Magíster en Educación con Mención en Formación Docente. Universidad Nacional de Entre Ríos. Profesora en Ciencias de la Educación. Universidad Nacional de Rosario. 


\title{
Palabras claves
}

Formas de Conocimiento - Enseñanza - Análisis de Clases.

\begin{abstract}
An account of this thesis defended in the year 2010, presents issues faced on a daily basis teachers of SC. of education working in teacher training institutes. Inquire about this topic motivation arises from the scant literature that deals with and deepens this modality. The intention then, was know as it gets in game knowledge in school classes. A priori, it went to the field with many feelings and assumptions that were broken down to research was spent in time and in its form. Thought on addressing what tours that occur in classes to achieve that knowledge arises within it, what kind of knowledge is it put into play, what happens with knowledge when he teaches it or proposed by the teacher when he teaches. Understand the ways of knowledge of teaching that were at stake in the classes taught by the professors of the Institute observed, also helped to analyze and reflect their own daily teaching work, and above all, recognize trails, to properly mediate the ability of students to understand and learn the contents that are "played" in the classroom.
\end{abstract}

\section{Key words}

Forms of knowledge - Teaching - Analysis classes.

\section{Presentación}

Interesarse en conocer cómo los sujetos se forman para ser docentes y como se aprende a enseñar, lleva a buscar la posibilidad de saber cuáles son las instancias que tienen mayor impacto o influencia en su formación. Esa relación estudiante-docente-conocimiento que se da en el ámbito áulico en un Instituto de Formación Docente, corresponde a una de esas instancias formativas importantes para el futuro profesorado, por eso el acercamiento a este objeto de estudio investigativo.

Para una mejor comprensión del lector, resulta conveniente presentar a esta investigación: primeramente abordando los problemas e interrogantes que se plantearon en la misma, luego mencionando a los objetivos generales y específicos y las opciones metodológicas, para luego continuar con el análisis del trabajo de campo y algunas aproximaciones de las categorías de análisis, y finalizar con las principales conclusiones.

\section{Problemas e interrogantes de la investigación}

Las clases se caracterizan por ser escenarios complejos, especialmente, cuando se pretende indagar qué ocurre con el conocimiento y cómo se organizan las relaciones entre los estudiantes, los docentes y el saber. 
Al adentrarse en esta complejidad, se empezaron a plantear algunos interrogantes que guiaron el desarrollo de la Tesina: ¿Cuáles son los recorridos, atajos, caminos, senderos que se producen en las clases y que se ponen en juego para lograr el conocimiento dentro de ella? ¿Cómo el docente presenta al contenido, en la clase escolar en un Instituto de Formación Docente? ¿Cómo incide la formación del docente en la presentación de su forma de enseñanza? y fundamentalmente ¿Cuál es la forma de conocimiento escolar que predominaría en las clases? ¿Qué tipo de conocimiento se pone en juego? ¿Qué sucede con el conocimiento cuando se lo enseña? y ¿Qué es lo que propone el docente cuando enseña?

\section{Objetivos generales y específicos de la investigación}

El objetivo general fue:

- Identificar las formas de conocimiento de la enseñanza que se ponen en juego en las clases dictadas por los profesores del Instituto de Formación Docente a observar.

Entre los objetivos específicos se observan:

a) Indagar en los factores que inciden en la elección de las formas de conocimiento que las docentes ponen en juego en sus clases.

b) Analizar las clases, en cuanto a como el conocimiento es presentado por las docentes: sus caminos, sus movimientos, sus jugadas, sus vericuetos.

\section{Acerca de las opciones metodológicas}

Para realizar esta investigación se partió de un enfoque metodológico cualitativo para tratar de lograr: a) un conocimiento como producción constructivistainterpretativa, ya que la interpretación es un proceso en el cual el investigador integra, reconstruye y presenta, en construcciones interpretativas, diversos indicadores producidos durante la investigación; y b) un proceso permanente de producción de conocimiento, donde los resultados fueron momentos parciales, que de manera continua se integraron dando lugar a nuevos interrogantes, abriendo nuevos caminos. Siguiendo a Achili (2001), se trató de realizar un continuo trabajo de problematización sobre el material recogido en el trabajo de campo, intentando llegar a formular otras problematizaciones en un movimiento espiralado, en el que cada vez se integraron más detalles, complejizándose la mirada. Fue crucial el análisis interpretativo y la contextualización del proceso de investigación, donde se trató de que la información sea sometida a análisis crítico, a contrastaciones, a triangulaciones. Esto fue llevando a ir armando nuevas construcciones y búsquedas de información, cada vez de un modo más focalizado y profundo. 
El trabajo de campo se realizó en un Instituto de Formación Docente de la ciudad de Rosario, con especialidad en Educación Física, donde se observaron cuatro clases consecutivas de tres profesoras durante los meses de Agosto, Septiembre, Octubre y Noviembre del año 2007. Las docentes fueron seleccionadas atendiendo a que dictaran espacios curriculares teóricos emparentados con la formación pedagógica de los estudiantes, como fueron Pedagogía, Teoría del Curriculum y Didáctica y el Trayecto de la Práctica. Para tratar de comprender el trabajo de campo, se utilizaron técnicas cualitativas que fueron: a-Observaciones de clases, b- Entrevistas semiestructuradas y c- Análisis de documentos. También para acercarse al contexto institucional se hizo un recorrido por algunas cuestiones históricas, de su política y de su diseño curricular.

\section{Análisis del trabajo de campo}

Para el mismo se desarrollaron gráficos en los que se intentó articular lo observado, con las categorías teóricas de análisis que estaban a priori y las que se fueron construyendo sobre la marcha. Las cuales fueron: el conocimiento escolar, la clase escolar y su relación con el conocimiento, la relación docenteconocimiento-clase escolar, la enseñanza y las formas de conocimiento en la enseñanza. También se hizo un recorrido sobre la formación docente, sus tradiciones, y los momentos de mayor impacto.

Se buscó entonces, en el proceso de análisis, desmenuzar el material y ampliar, contextualizar, y producir descripciones cada vez más integrativas, rastreando indicios. Se intentó interpretar, intentando analizar críticamente, contrastar, triangular lo que se consideró de validez para la realización del objetivo de la investigación. Por eso, y fruto de estas cuestiones, encontramos en este entrecruzamiento, los siguientes puntos salientes: a) La comunicación del conocimiento por parte del docente y su mediación, b) El análisis del conocimiento en el aula, c) Las formas de conocimiento en la enseñanza en las clases propuestas por las docentes, d) La biografía escolar, su incidencia y e) La subjetividad en la relación docente-conocimiento-clase escolar.

\section{Conclusiones}

A partir entonces, de estos ítems y de querer encontrar "el eco del original", partiendo de la práctica para ir a la teoría y viceversa; se puede ahora, mencionar las conclusiones generales sobre este trabajo que seguramente no son finales, sino que abren nuevas propuestas para entender el complejo mundo del aula y la relación con el conocimiento.

En primer lugar, habría una estructura, una arquitectura, un diseño o un camino, que posibilitaría a que el docente presente el conocimiento en el aula de una forma y no de otra (aunque esté atravesado por diferentes formas de presentarlo, que tal vez sea una forma de hacerlo), y que este camino estaría 
compuesto por: las estrategias metodológicas que emplea, los contenidos elegidos para enseñar, su discurso, su ideología, su subjetividad, los conocimientos que tiene sobre su materia, su didáctica, sus experiencias (laborales, personales etc.), su historia personal, su biografía escolar, su relación y conocimiento del contexto (el grupo escolar, lo socio cultural, la institución, sus colegas). Estos componentes dan como resultado una estructura en el sujeto, la cual, se puede denominar como un habitus particular: un habitus de enseñanza o habitus de enseñar, que llevaría al docente a plantear su forma de conocimiento de la enseñanza de ese modo particular que lo hace, y no de otro. Gracias a esta "estructura estructurante", a esa "gramática generativa" de prácticas, los docentes son capaces de enfrentarse a su trabajo. Se construye y luego se estabiliza un esquema que permite, progresivamente, realizar las prácticas de la enseñanza. Sería entonces un esquema de pensamiento, de percepción, evaluación y de acción con respecto a la forma de como los docentes ponen en juego el conocimiento en la enseñanza, donde el motor y centro sería su habitus de enseñanza. Esto es, los docentes pondrían en juego ese habitus de enseñanza en particular, porque el mismo se pone de manifiesto en las acciones humanas, y el enseñar conocimientos, es la acción que hacen fundamentalmente en el aula los docentes. Ese habitus sería el pilar y la base de las formas del conocimiento de enseñanza que el profesor propone en las clases. Tal vez se puede decir que actuaría como mediador entre los saberes y las situaciones que exigen una acción en el docente. Tal vez se lo pueda asociar al habitus profesional, que son los esquemas adquiridos en la historia que se incorporan y son puestos en acto en las prácticas cotidianas. Pero este habitus (el de enseñar o enseñanza) tal vez al tener su raíz en una secuencia donde el docente ingresa al ámbito escolar desde muy niño, tendría una particularidad especial con respecto a éste.

En segundo lugar, no habría una forma de conocimiento que prevalece en las clases observadas, una misma docente puede tomar las tres formas que plantea Edwards (1992) -conocimiento situado, operacional y tópico-, y puede fluctuar entre las tres en sus prácticas de enseñanza (1).

Lo que sí se observa es que en esta instancia de Formación Docente Inicial, se apuntaría a la comprensión, y en especial a la comprensión del "ser docente", o de las cuestiones importantes que un docente debería tener para hacer su trabajo cotidiano. Tal vez por haber observado materias llamadas "pedagógicas", todos los docentes observados iban permanentemente al ejemplo práctico de situaciones del trabajo cotidiano docente. Se cree entonces, que en este ámbito, podría existir una nueva forma de conocimiento en la enseñanza adjuntándola a las tres que Verónica Edwards (1992) describe; y que sería el conocimiento comprensivo. Éste no se estructuraría básicamente en torno al interés de conocer una situación, ni a la repetición mecánica del saber, ni a la posibilidad de utilizarlo como operación, pero sí a la comprensión de los 
contenidos. No, necesariamente, ligaría al sujeto con su situación, la cual está formada por usos y costumbres actuales de procesos construidos históricamente y desde una posición de clase en la sociedad, pero sí tendría que articular, comparar, escuchar, comprender los saberes que el docente le presenta de diferentes maneras. Esta forma de conocimiento que se plantea, sería la que prevalece en las prácticas de la enseñanza observadas.

Las tres docentes observadas tienen su objetivo en la comprensión estudiantil; en que los jóvenes puedan comprender los contenidos que se juegan, al decir de Burbules (1999), en la clase. El objetivo de las docentes es que el contenido se comprenda, que lo que pase en la clase se comprenda, y para ello, pueden usarse las instancias que ellas, desde su subjetividad (y porque no decir de su habitus de enseñar), consideran mejores: estrategias metodológicas, recursos materiales, actividades, preguntas, organización de la clase, evaluación, selección de contenidos etc. Se cree que las docentes observadas ponen en juego las diversas formas de conocimiento en la enseñanza, pero fundamentalmente apuntarían a la comprensión.

Para finalizar estas conclusiones se puede decir que esta investigación fue también, para los autores, un disparador de reflexiones e interrogaciones propias: ¿Para qué pasar por la clase? ¿Qué se "esta haciendo" con la clase y el conocimiento? ¿Cuál es el objetivo del docente con respecto a esa relación? ¿Se los tiene en realidad, o se sacan contenidos como "conejos de la galera" para llegar con el programa? ¿Se reestructuran las clases para que los estudiantes comprendan mejor los contenidos?, ¿En las mismas, se ponen en juego conocimientos que les sirva a los estudiantes para ser buenos profesores? ¿Cómo se pone en juego el conocimiento?, ¿Qué y cómo se enseña? ¿Los docentes reflexionan sobre lo que hacen en la clase para poder transformar sus prácticas?

Como vemos, se abren nuevos interrogantes a partir de este trabajo, que sin dudas no encuentran respuestas concisas en los autores; y que apuntan a reflexionar sobre las propias prácticas de la enseñanza, para poder transformarlas y mejorarlas.

\section{Nota Bibliográfica}

(1) No afirmamos que hay un cambio de concepción de conocimiento, sí de la forma de abordarlo en la clase por el/la docente, creemos que se "juega" (conciente o inconscientemente) con las tres formas de conocimiento en la enseñanza, es decir, una misma docente en el recorrido de la clase o en diferentes clases puede presentar las tres formas que plantea Edwards, por eso decimos que no prevalece una forma, ni niveles de concepción de conocimiento, simplemente que se pueden "tomar" las tres y que las tres pueden hasta fluctuar en una misma clase 


\section{Referencias Bibliográficas}

- Achili, E. (2001) "Metodología y técnicas de investigación”. Modulo III. Material de cátedra. Postítulo en Investigación Educativa a distancia. Universidad Nacional de Córdoba.

- Burbules, N. (1999) "El diálogo en la enseñanza. Teoría y práctica". Buenos Aires: Amorrortu.

- $\quad$ Arnay, J., Rodrigo, M.J. (1997) “La construcción del conocimiento escolar”. Barcelona: Paidos.

- Cullen, C. (1997). "Las razones de educar". Buenos Aires: Paidos.

- Camilioni, A. Davini, M. C., Eldestein, G. Litwin, E. Souto M. y Barco, S. (1999). "Corrientes didácticas contemporáneas". Buenos Aires: Paidós.

- Camilioni A. (2007) "El saber didáctico": Buenos Aires: Paidos

- Davini, M. C. (1995). "La formación docente en cuestión: política y pedagogía”. Buenos Aires: Paidós.

- Diker F. y Terigi G. (1997). "La formación de maestros y profesores. Hoja de ruta." Buenos Aires: Paidos.

- $\quad$ Edwards V. (1992) "Los sujetos y la construcción social del conocimiento escolar en primaria. Un estudio etnográfico". Tesis de maestría. Mimeografiado Instituto Politécnico Nacional. México.

- Entel, A. (1988). "Escuela y conocimiento” Buenos Aires: Miño y Davila Editores.

- $\quad$ Ferry, G. (1990). "El trayecto de la formación”. México: Paidós.

- Frigerio, G. Poggi, M., Korinfeld E. (comps.) (1999). "Construyendo un saber sobre el interior de la escuela". Buenos Aires: Novedades Educativas.

- Gimeno Sacristán J. y Perez Gomez A. (1997) "Comprender y transformar la enseñanza" Madrid: Morata.

- Geertz, C. 1973. "La interpretación de las culturas" Barcelona: Gedisa.

- $\quad$ Guber, R. (1991) “El salvaje metropolitano”. Buenos Aires: Legasa.

- Jackson, Ph.(1998). "La vida en las aulas". Madrid: Morata.

- Lipman, M. (1998). "Pensamiento complejo y educación". Madrid: Ediciones de la Torre.

- Litwin, E. (1997). "Las configuraciones didácticas". Buenos Aires: Paidós

- $\quad$ Litwin, E. (2008). "El oficio de enseñar. Condiciones y contextos”. Buenos Aires: Paidós.

- Marturana, H. (1997). "La Objetividad. Un argumento para obligar". Santiago de Chile: Dolmen.

- Morin, E. (1989). “Sujeto y Objeto”, en Briceño León R. y Wagner E. (comp.). "Las Ciencias de lo Humano: Homenaje internacional a Jeannette Abouhamad". Caracas: Fondo Editorial Acta Científica Venezolana.

- Morin, E. (1994). "Introducción al Pensamiento Complejo": Barcelona. Gedisa.

- Perrenaud Ph, Altet, M, Paquay, L, Charlier E. (Coords.) (2005). "La formación profesional de los maestros". México: Fondo de Cultura Económica.

- Sanjurjo, L. (2002). "La formación práctica de los docentes. Reflexión y acción en el aula”. Rosario: Homo Sapiens.

- Sanjurjo, L. (2003) "Volver a pensar la clase": Rosario: Homo Sapiens.

- Sanjurjo, L. (Comps). (2009). "Los dispositivos para la formación en las prácticas profesionales". Rosario: Homo Sapiens.

- Tadeu da Silva, T. (1995). "Escuela conocimiento y currículo. Ensayos críticos”. Buenos. Aires: Miño y Davila Editores. 